\title{
Routing regardless of Network Stability
}

\author{
Bundit Laekhanukit* Adrian Vetta ${ }^{\dagger} \quad$ Gordon Wilfong ${ }^{\ddagger}$
}

November 8, 2018

\begin{abstract}
How effective are interdomain routing protocols, such as the Border Gateway Protocol, at routing packets? Theoretical analyses have attempted to answer this question by ignoring the packets and instead focusing upon protocol stability. To study stability, it suffices to model only the control plane (which determines the routing graph) - an approach taken in the Stable Paths Problem. To analyse packet routing requires modelling the interactions between the control plane and the forwarding plane (which determines where packets are forwarded), and our first contribution is to introduce such a model. We then examine the effectiveness of packet routing in this model for the broad class next-hop preferences with filtering. Here each node $v$ has a filtering list $\mathcal{D}(v)$ consisting of nodes it does not want its packets to route through. Acceptable paths (those that avoid nodes in the filtering list) are ranked according to the next-hop, that is, the neighbour of $v$ that the path begins with. On the negative side, we present a strong inapproximability result. For filtering lists of cardinality at most one, given a network in which an equilibrium is guaranteed to exist, it is NP-hard to approximate the maximum number of packets that can be routed to within a factor of $n^{1-\epsilon}$, for any constant $\epsilon>0$. On the positive side, we give algorithms to show that in two fundamental cases every packet will eventually route with probability one. The first case is when each node's filtering list contains only itself, that is, $\mathcal{D}(v)=\{v\}$; this is the fundamental case in which a node does not want its packets to cycle. Moreover, with positive probability every packet will be routed before the control plane reaches an equilibrium. The second case is when all the filtering lists are empty, that is, $\mathcal{D}(v)=\emptyset$. Thus, with probability one packets will route even when the nodes do not care if their packets cycle! Furthermore, with probability one every packet will route even when the control plane has no equilibrium at all. To our knowledge, these are the first results to guarantee the possibility that all packets get routed without stability. These positive results are tight - for the general case of filtering lists of cardinality one, it is not possible to ensure that every packet will eventually route.
\end{abstract}

\section{Introduction}

In the Stable Paths Problem (SPP) [5], we are given a directed graph $G=(V, A)$ and a sink (or destination) node $r$. Furthermore, each node $v$ has a ranked list of some of its paths to $r$. The

\footnotetext{
* School of Computer Science, McGill University. Supported by a Dr. and Mrs. Milton Leong Fellowship and by NSERC grant 288334. Email: blaekh@cs.mcgill.ca

${ }^{\dagger}$ Department of Mathematics and Statistics and School of Computer Science, McGill University. Supported in part by NSERC grants 288334 and 429598 . Email: vetta@math.mcgill.ca

${ }^{\ddagger}$ Bell Laboratories. Email: gtw@research.bell-labs.com
} 
lowest ranked entry in the list is the "empty path" 1, paths that are not ranked are considered unsatisfactory. This preference list is called $v$ 's list of acceptable paths. A set of paths, one path $\mathcal{P}(v)$ from each node $v$ 's list of acceptable paths, is termed stable if

(i) they are consistent: if $u \in \mathcal{P}(v)$, then $\mathcal{P}(u)$ must be the subpath of $\mathcal{P}(v)$ beginning at $u$, and

(ii) they form an equilibrium: for each node $v, \mathcal{P}(v)$ is the path ranked highest by $v$ of the form $v \mathcal{P}(w)$ where $w$ is a neighbour of $v$.

The stable paths problem asks whether a stable set of paths exists in the network. The SPP has risen to prominence as it is viewed as a static description of the problem that the Border Gateway Protocol (BGP) is trying dynamically to solve. BGP can be thought of as trying to find a set of stable routes to $r$ so that routers can use these routes to send packets to $r$.

Due to the importance of BGP, both practical and theoretical aspects of the SPP have been studied in great depth. In the main text, to avoid overloading the reader with practical technicalities, we focus on the combinatorial aspects of packet routing; in the Appendix we discuss the technical aspects and present a motivating sample of the vast literature on BGP. Two observations concerning the SPP, though, are pertinent here and motivate our work:

(1) Even if a stable solution exists, the routing tree induced by a consistent set of paths might not be spanning. Hence, a stable solution may not actually correspond to a functioning network - there may be isolated nodes that cannot route packets to the sink! Disconnectivities arise because nodes may prefer the empty-path to any of the paths proffered by its neighbours; for example, a node might not trust certain nodes to handle its packets securely or in a timely fashion, so it may reject routes traversing such unreliable domains. This problem of nonspanning routing trees has quite recently been studied in the context of a version of BGP called iBGP [19]. In Section 3, we show that non-connectivity is a very serious problem (at least, from the theoretical side) by presenting an $n^{1-\epsilon}$ hardness result for the combinatorial problem of finding a maximum cardinality stable subtree.

(2) The SPP says nothing about the dynamic behaviour of BGP. Stable routings are significant for many practical reasons (e.g., network operators want to know the routes their packets are taking), but while BGP is operating at the control plane level, packets are being sent at the forwarding plane level without waiting for stability (if, indeed, stability is ever achieved). Thus, it is important to study network performance in the dynamic case. For example, what happens to the packets whilst a network is unstable? This is the main focus of our paper: to investigate packet routing under network dynamics.

Towards this goal, we define a distributed protocol, inspired by BGP, that stops making changes to the routing graph (i.e., becomes stable) if it achieves a stable solution to the underlying instance of SPP. The current routing graph itself is determined by the control plane but the movement of packets is determined by the forwarding plane. Thus, our distributed protocol provides a framework under which the control and forwarding planes interact; essentially, this primarily means that we need to understand the relative speeds at which links change and packets move.

Given this model, we analyse the resulting trajectory of packets. In a stable solution, a node in the stable tree containing the sink would have its packets route whereas an isolated node would

\footnotetext{
${ }^{1}$ Clearly, the empty path is not a real path to the sink; we call it a path for clarity of exposition.
} 
not. For unstable networks, or for stable networks that have not converged, things are much more complicated. Here the routes selected by nodes are changing over time and, as we shall see, this may cause the packets to cycle. If packets can cycle, then keeping track of them is highly non-trivial. Our main results, however, are that for two fundamental classes of preference functions (i.e., two ways of defining acceptable paths and their rankings) all packets will route with probability one in our model. That is, there is an execution of our distributed protocol such that every packet in the network will reach the destination (albeit, possibly, slowly) even in instances where the network has no stable solution. (Note that we are ignoring the fact that in BGP packets typically have a time-to-live attribute meaning that after traversing a fixed number of nodes the packet will be dropped.) Furthermore, when the network does have a stable solution, we are able to guarantee packet routing even before the time when the network converges.

These positive results on the routing rate are to our knowledge, the first results to guarantee the possibility of packet routing without stability. The results are also tight in the sense that, for any more expressive class of preference function, our hardness results show that guaranteeing that all packets eventually route is not possible - thus, packets must be lost.

\section{The Model and Results}

We represent a network by a directed graph $G=(V, A)$ on $n$ nodes. The destination node in the network is denoted by a distinguished node $r$ called a sink node. We assume that, for every node $v \in V$, there is at least one directed path in $G$ from $v$ to the sink $r$, and that the sink $r$ has no outgoing arc. At any point in time $t$, each node $v$ chooses at most one of its out-neighbours $w$ as its chosen next-hop; thus, $v$ selects one arc $(v, w)$ or selects none. These arcs form a routing graph $\mathcal{R}_{t}$, each component of which is a 1-arborescence, an in-arborescenc $\&^{2} T$ plus possibly one arc $(v, w)$ emanating from the root $v$ of $T$; for example, $T$ and $T \cup\{(v, w)\}$ are both 1-arborescences. (If the root of a component does select a neighbour, then that component contains a unique cycle.) When the context is clear, for clarity of exposition, we abuse the term tree to mean a 1-arborescence, and we use the term forest to mean a set of trees. A component (tree) in a routing graph is called a sink-component if it has the sink $r$ as a root; all other components are called non-sink components.

Each node selects its outgoing arc according to its preference list of acceptable paths. We examine the case where these lists can be generated using two of the most common preference criteria in practice: next-hop preferences and filtering. For next-hop preferences, each node $v \in V$ has a ranking on its out-neighbours, nodes $w$ such that $(v, w) \in A$. We say that $w$ is the $k$-th choice of $v$ if $w$ is an out-neighbour of $v$ with the $k$-th rank. For $k=1,2, \ldots, n$, we define a set of arcs $A_{k}$ to be such that $(v, w) \in A_{k}$ if $w$ is the $k$-th choice of $v$, i.e., $A_{k}$ is the set of the $k$-th choice arcs. Thus, $A_{1}, A_{2}, \ldots, A_{n}$ partition the set of $\operatorname{arcs} A$, i.e., $A=A_{1} \cup A_{2} \cup \ldots A_{n}$. We call the entire graph $G=(V, A)$ an all-choice graph. A filtering list, $\mathcal{D}(v)$, is a set of nodes that $v$ never wants its packets to route through. We allow nodes to use filters and otherwise rank routes via next-hop preferences, namely next-hop preferences with filtering.

To be able to apply these preferences, each node $v \in V$ is also associated with a path $\mathcal{P}(v)$, called $v$ 's routing path. The routing path $\mathcal{P}(v)$ may not be the same as an actual $v, r$-path in the routing graph. We say that a routing path $\mathcal{P}(v)$ is consistent if $\mathcal{P}(v)$ is a $v, r$-path in the routing graph; otherwise, we say that $\mathcal{P}(v)$ is inconsistent. Similarly, we say that a node $v$ is consistent if

\footnotetext{
${ }^{2}$ An in-arborescence is a graph $T$ such that the underlying undirected graph is a tree and every node has a unique path to a root node.
} 
its routing path $\mathcal{P}(v)$ is consistent; otherwise, we say that $v$ is inconsistent. A node $v$ is clear if the routing path $\mathcal{P}(v) \neq \emptyset$, i.e., $v$ (believes it) has a path to the sink; otherwise, $v$ is opaque. We say that a node $w$ is valid for $v$ or is a valid choice for $v$ if $w$ is clear and $\mathcal{P}(w)$ contains no nodes in the filtering list $\mathcal{D}(w)$. If $w$ is a valid choice for $v$, and $v$ prefers $w$ to all other valid choices, then we say that $w$ is the best valid choice of $v$. A basic step in the dynamic behaviour of BGP is that, at any time $t$, some subset $V_{t}$ of nodes is activated meaning that every node $v \in V_{t}$ chooses the highest ranked acceptable path $\mathcal{P}(v)$ that is consistent with one of its neighbours' routing paths at time $t-1$. The routing graph $\mathcal{R}_{t}$ consists of the first arc in each routing path at time $t$.

Protocol variations result from such things as restricting $V_{t}$ so that $\left|V_{t}\right|=1$, specifying the relative rates that nodes are chosen to be activated and allowing other computations to occur between these basic steps. In our protocol, we assume that activation orderings are fair in that each node activates exactly once in each time period - a round - the actual ordering however may differ in each round. While our protocol is not intended to model exactly the behaviour of BGP, we tried to let BGP inspire our choices and to capture the essential coordination problem that makes successful dynamic routing hard. Again, a detailed discussion on these issues and on the importance of a fairness-type criteria is deferred to the Appendix.
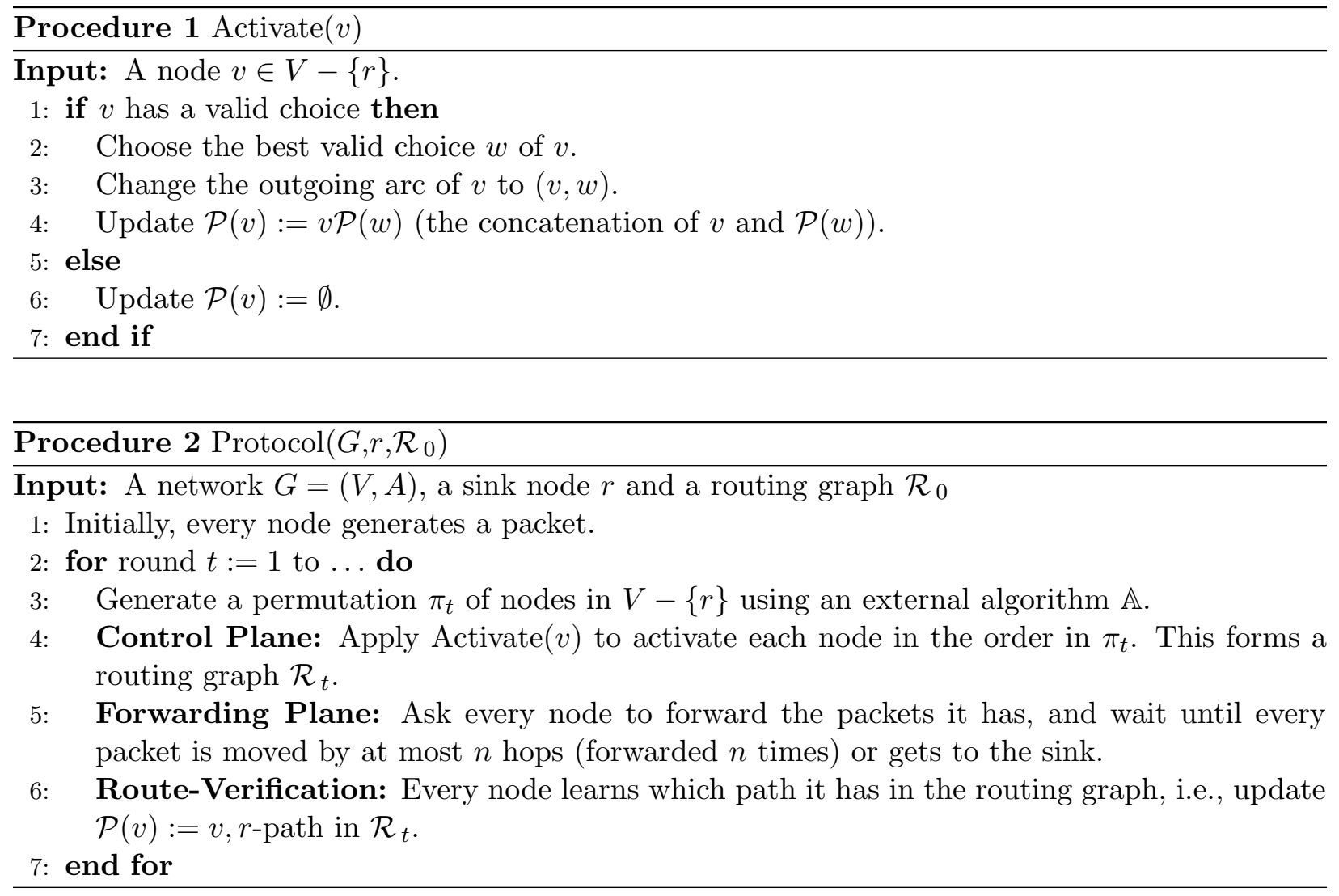

This entire mechanism can thus be described using two algorithms as follows. Once activated, a node $v$ updates its routing path $\mathcal{P}(v)$ using the algorithm in Procedure 1. The generic protocol is described in Procedure 2. This requires an external algorithm $\mathbb{A}$ which acts as a scheduler that generates a permutation - an order in which nodes will be activated in each round. We will assume that these permutations are independent and randomly generated. Our subsequent routing 
guarantees will be derived by showing the existence of specific permutations that ensure all packets route. These permutations are different in each of our models, which differ only in the filtering lists. We remark that our model is incorporated with a route-verification step, but this is not a feature of BGP (see the Appendix for a discussion).

With the model defined, we examine the efficiency of packet routing for the three cases of next-hop preferences with filtering:

- General Filtering. The general case where the filtering list $\mathcal{D}(v)$ of any node $v$ can be an arbitrary subset of nodes.

- Not me! The subcase where the filtering list of node $v$ consists only of itself, $\mathcal{D}(v)=\{v\}$. Thus, a node does not want a path through itself, but otherwise has no nodes it wishes to avoid.

- Anything Goes! The case where every filtering list is empty, $\mathcal{D}(v)=\emptyset$. Thus a node does not even mind if its packets cycle back through it!

\subsection{Our Results.}

We partition our analyses based upon the types of filtering lists. Our first result is a strong hardness result presented in Section 3. Not only can it be hard to determine if every packet can be routed but the maximum number of packets that can be routed cannot be approximated well even if the network can reach equilibrium. Specifically,

Theorem 1. For filtering lists of cardinality at most one, it is NP-hard to approximate the maximum cardinality stable subtree to within a factor of $n^{1-\epsilon}$, for any constant $\epsilon>0$.

Corollary 2. For filtering lists of cardinality at most one, given a network in which an equilibrium is guaranteed to exist, it is NP-hard to approximate the maximum number of packets that can be routed to within a factor of $n^{1-\epsilon}$, for any constant $\epsilon>0$.

However, for its natural subcase where the filtering list of a node consists only of itself (that is, a node does not want to route via a cycle!), we obtain a positive result in Section 5 .

Theorem 3. If the filtering list of a node consists only of itself, then an equilibrium can be obtained in $n$ rounds. Moreover, every packet will be routed in $\frac{n}{3}$ rounds, that is, before stability is obtained!

Interestingly, we can route every packet in the case $\mathcal{D}(v)=\emptyset$ for all $v \in V$; see Section 4 . Thus, even if nodes do not care whether their packets cycle, the packets still get through!

Theorem 4. If the filtering list is empty then every packet can be routed in 4 rounds, even when the network has no equilibrium.

Theorems 3 and 4 are the first theoretical results showing that packet routing can be done in the absence of stability. For example, every packet will be routed even in the presence of dispute wheels [5]. Indeed, packets will be routed even if some nodes never actually have paths to the sink. Note that when we say that every packet will route with probability one we mean that, assuming permutations are drawn at random, we will eventually get a fair activation sequence that routes every packet. It is a nice open problem to obtain high probability guarantees for fast packet routing under such an assumption. 


\section{General Filtering.}

Here we consider hardness results for packet routing with general filtering lists. As discussed, traditionally the theory community has focused upon the stability of $\mathcal{R}$ - the routing graph is stable if every node is selecting their best valid neighbour (and is consistent). For example, there are numerous intractability results regarding whether a network has an equilibrium; e.g., see [6, 2]. However, notice that the routing graph may be stable even if it is not spanning! There may be singleton nodes that prefer to stay disconnected rather than take any of the offered routes. Thus, regardless of issues such as existence and convergence, an equilibrium may not even route the packets. This can be particularly problematic when the nodes use filters. Consider our problem of maximising the number of nodes that can route packets successfully. We show that this cannot be approximated to within a factor of $n^{1-\epsilon}$, for any $\epsilon>0$ unless $\mathrm{P}=\mathrm{NP}$. The proof is based solely upon a control plane hardness result: it is NP-hard to approximate the maximum cardinality stable tree to within a factor of $n^{1-\epsilon}$. Thus, even if equilibria exist, it is hard to determine if there is one in which the sink-component (the component of $\mathcal{R}$ containing the sink) is large.

Formally, in the maximum cardinality stable tree problem, we are given a directed graph $G=$ $(V, E)$ and a sink node $r$; each node $v \in V$ has a ranking of its neighbours and has a filtering list $\mathcal{D}(v)$. Given a tree (arborescence) $T \subseteq G$, we say that a node $v$ is valid for a node $u$ if $(u, v) \in E$ and a $v, r$-path in $T$ does not contain any node of $\mathcal{D}(v)$. We say that $T$ is stable if, for every arc $(u, v)$ of $T$, we have that $v$ is valid for $u$, and $u$ prefers $v$ to any of its neighbours in $G$ that are valid for $u$ (w.r.t. $T$ ). Our goal is to find a stable tree (sink-component) with the maximum number of nodes. We will show that even when $|\mathcal{D}(v)|=1$ for all nodes $v \in V$, the maximum-size stable tree problem cannot be approximated to within a factor of $n^{1-\epsilon}$, for any constant $\epsilon>0$, unless $\mathrm{P}=\mathrm{NP}$.

The proof is based on the hardness of 3SAT [10]: given a CNF-formula on $N$ variables and $M$ clauses, it is NP-hard to determine whether there is an assignment satisfying all the clauses. Take an instance of 3SAT with $N$ variables, $x_{1}, x_{2}, \ldots, x_{N}$ and $M$ clauses $C_{1}, C_{2}, \ldots, C_{M}$. We now create a network $G=(V, A)$ using the following gadgets:

- Variable-Gadget: For each variable $x_{i}$, we have a gadget $H\left(x_{i}\right)$ with four nodes $a_{i}, u_{i}^{T}, u_{i}^{F}, b_{i}$. The nodes $u_{i}^{T}$ and $u_{i}^{F}$ have first-choice $\operatorname{arcs}\left(u_{i}^{T}, a_{i}\right),\left(u_{i}^{F}, a_{i}\right)$ and second-choice $\operatorname{arcs}\left(u_{i}^{T}, b_{i}\right)$, $\left(u_{i}^{F}, b_{i}\right)$. The node $a_{i}$ has two $\operatorname{arcs}\left(a_{i}, u_{i}^{T}\right)$ and $\left(a_{i}, u_{i}^{F}\right)$; the ranking of these arcs can be arbitrary. Each node in this gadget has itself in the filtering list, i.e., $\mathcal{D}(v)=\{v\}$ for all nodes $v$ in $H\left(x_{i}\right)$.

- Clause-Gadget: For each clause $C_{j}$ with three variables $x_{i(1)}, x_{i(2)}, x_{i(3)}$, we have a gadget $Q\left(C_{j}\right)$. The gadget $Q\left(C_{j}\right)$ has four nodes $s_{j}, q_{1, j}, q_{2, j}, q_{3, j}, t_{j}$. The nodes $q_{1, j}, q_{2, j}, q_{3, j}$ have first-choice $\operatorname{arcs}\left(q_{1, j}, t_{j}\right),\left(q_{2, j}, t_{j}\right),\left(q_{3, j}, t_{j}\right)$. The node $s_{j}$ has three $\operatorname{arcs}\left(s_{j}, q_{1, j}\right),\left(s_{j}, q_{2, j}\right)$, $\left(s_{j}, q_{3, j}\right)$; the ranking of these arcs can be arbitrary, so we may assume that $\left(s_{j}, q_{z, j}\right)$ is a $z$ th-choice arc. Define the filtering list of $s_{j}$ and $t_{j}$ as $\mathcal{D}\left(s_{j}\right)=\left\{s_{j}\right\}$ and $\mathcal{D}\left(t_{j}\right)=\left\{d_{0}\right\}$. (The node $d_{0}$ will be defined later.) For $z=1,2,3$, let $u_{i(z)}^{T}$ and $u_{i(z)}^{F}$ be nodes in the corresponding Variable-Gadget $H\left(x_{i(z)}\right)$. The node $q_{z, j}$ has a filtering list $\mathcal{D}\left(q_{z, j}\right)=\left\{u_{i(z)}^{T}\right\}$, if assigning $x_{i(z)}=$ False satisfies the clause $C_{j}$; otherwise, $\mathcal{D}\left(q_{z, j}\right)=\left\{u_{i(z)}^{F}\right\}$.

To build $G$, we first add a sink node $r$ and a dummy "sink" $d_{0}$. We then connect $d_{0}$ to $r$ by a first-choice arc $\left(d_{0}, r\right)$. We number the Variable-Gadgets and Clause-Gadgets in any order. Then we add a first-choice arc from the node $a_{1}$ of the first Variable-Gadget $H\left(x_{1}\right)$ to the sink $r$. For $i=2,3, \ldots, N$, we add a first-choice arc $\left(b_{i}, a_{i-1}\right)$ joining gadgets $H\left(x_{i-1}\right)$ and $H\left(x_{i}\right)$. We join the 
last Variable-Gadget $H\left(x_{N}\right)$ and the first Clause-Gadget $Q\left(C_{1}\right)$ by a first-choice arc $\left(t_{1}, a_{N}\right)$. For $j=2,3, \ldots, M$, we add a first-choice arc $\left(t_{j}, s_{j-1}\right)$ joining gadgets $Q\left(C_{j-1}\right)$ and $Q\left(C_{j}\right)$. This forms a line of gadgets. Then, for each node $q_{z, j}$ of each Clause-Gadget $Q\left(C_{j}\right)$, we add a second-choice $\operatorname{arc}\left(q_{z, j}, d_{0}\right)$ joining $q_{z, j}$ to the dummy sink $d_{0}$. Finally, we add $L$ padding nodes $d_{1}, d_{2}, \ldots, d_{L}$ and join each node $d_{i}$, for $i=1,2, \ldots, L$, to the last Clause-Gadget $Q\left(C_{M}\right)$ by a first-choice arc $\left(d_{i}, s_{M}\right)$; the filtering list of each node $d_{i}$ is $\mathcal{D}\left(d_{i}\right)=\left\{d_{0}\right\}$, for all $i=0,1, \ldots, L$. The parameter $L$ can be any positive integer depending on a given parameter. Observe that the number of nodes in the graph $G$ is $4 N+5 M+L+2$, and $|\mathcal{D}(v)|=1$ for all nodes $v$ of $G$. The reduction is illustrated in Figure 1.

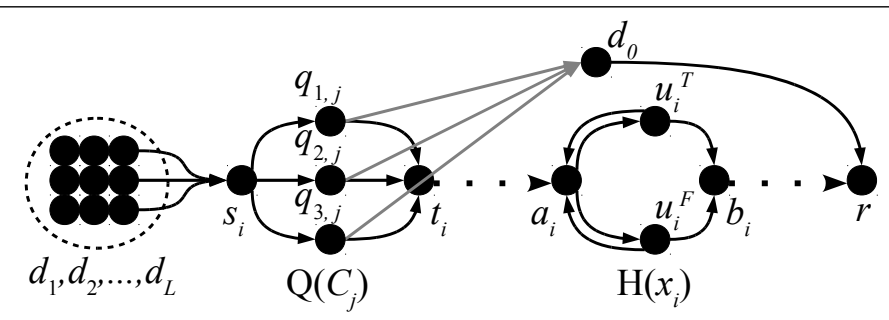

Figure 1: The hardness construction.

The correctness of the reduction is proven in the next theorem.

Theorem 5. For any constant $\epsilon>0$, given an instance of the maximum-size stable tree problems with a directed graph $G$ on $n$ nodes and filtering lists of cardinality $|\mathcal{D}(v)|=1$ for all nodes $v$, it is NP-hard to distinguish between the following two cases of the maximum cardinality stable tree problem.

- Yes-Instance: The graph $G$ has a stable tree spanning all the nodes.

- No-Instance: The graph $G$ has no stable tree spanning $n^{\epsilon}$ nodes.

Proof. We apply the above reduction from 3SAT with a parameter $L=J^{1 / \epsilon}-J$, where $J=$ $4 n+5 m+2$. Thus, the graph $G$ has $n=J^{1 / \epsilon}$ nodes and has $n^{\epsilon}=J$ non-padding nodes.

First, we show that there is a one-to-one mapping between choices of each Variable-Gadget $H\left(x_{i}\right)$ and an assignment of $x_{i}$. Consider any Variable-Gadget $H\left(x_{i}\right)$. To connect to the next gadget, nodes $u_{i}^{T}$ and $u_{i}^{F}$ of $H\left(x_{i}\right)$ must choose at least one second-choice arc. However, in a stable tree, they cannot choose both second-choice $\operatorname{arcs}\left(u_{i}^{T}, b_{i}\right)$ and $\left(u_{i}^{F}, b_{i}\right)$; otherwise, $u_{i}^{T}$ or $u_{i}^{F}$ would prefer to choose the node $a_{i}$. Thus, the gadget $G\left(x_{i}\right)$ must choose either arcs

$$
\text { (1) }\left(u_{i}^{T}, b_{i}\right),\left(u_{i}^{F}, a_{i}\right),\left(a_{i}, u_{i}^{T}\right) \quad \text { or } \quad(2)\left(u_{i}^{F}, b_{i}\right),\left(u_{i}^{T}, a_{i}\right),\left(a_{i}, u_{i}^{F}\right) .
$$

These two cases correspond to the the assignments $x_{i}=$ True and $x_{i}=$ False, respectively. Thus, there is a one-to-one mapping between the choices of gadget $H\left(x_{i}\right)$ in the stable tree and the assignment of $x_{i}$. We refer to each of these two alternatives as an assignment of $x_{i}$.

Now, we prove the correctness of the reduction.

Yes-Instance: Suppose there is an assignment satisfying all the clauses. Then there is a stable tree $T$ corresponding to such an assignment. To see this, within Variable-Gadget we select arcs in accordance with the assignment as detailed above. We also choose the arc $\left(d_{0}, r\right)$ and all the 
horizontal arcs connecting adjacent gadgets in the line (or from the first Variable-Gadget to the sink $r)$. For each Clause-Gadget $Q\left(C_{j}\right)$ and each $z=1,2,3$, we choose the first-choice arc $\left(q_{z, j}, t_{j}\right)$ if the assignment to $x_{i(z)}$ satisfies $C_{j}$; otherwise, we choose the second-choice arc $\left(q_{z, j}, d_{0}\right)$. For the node $s_{j}$ of $Q\left(C_{j}\right)$, we choose an arc $\left(s_{j}, q_{z, j}\right)$, where $z$ is the smallest number such that the assignment to $x_{i(z)}$ satisfies $C_{j}$ (i.e., $q_{z, j}$ chooses $t_{j}$ ); since the given assignment satisfies all the clauses, $s_{j}$ has at least one valid choice. Now, we have that the node $s_{M}$ of the last Clause-Gadget $Q\left(C_{j}\right)$ has a path $\mathcal{P}\left(s_{M}\right)$ to the sink $r$ that does not contain the dummy sink $d_{0}$. Thus, every padding node can choose $s_{M}$ and, therefore, is in the stable tree $T$. This implies that $T$ spans all the nodes.

No-Instance: Suppose there is no assignment satisfying all the clauses. Let $T$ be any stable tree of $G$. As in the previous discussion, the choices of nodes in Variable-Gadgets correspond to the assignment of variables of 3SAT.

Consider any Clause-Gadget $Q\left(C_{j}\right)$. Since $\mathcal{D}\left(s_{j}\right)=\left\{d_{0}\right\}$, the node $s_{j}$ of $Q\left(C_{j}\right)$ has a path to the sink $r$ only if

(1) a $t_{j}, r$-path $\mathcal{P}\left(t_{j}\right)$ in $T$ does not contain the dummy sink $d_{0}$, and

(2) one of $q_{1, j}, q_{2, j}, q_{3, j}$ chooses $t_{j}$.

These two conditions hold only if $T$ corresponds to an assignment satisfying $C_{j}$. To see this, suppose the first condition holds. Then $\mathcal{P}\left(t_{j}\right)$ has to visit either $v_{i}^{F}$ or $v_{i}^{T}$ of every Variable-Gadget $H\left(x_{i}\right)$, depending on the assignment of $x_{i}$. Thus, by the construction of $\mathcal{D}\left(q_{z, j}\right), t_{j}$ is valid for $q_{z, j}$ only if the assignment to $x_{i(z)}$ satisfies $C_{j}$. Since there is no assignment satisfying all the clauses, a node $s_{\ell}$ of some Clause-Gadget $Q\left(C_{\ell}\right)$ is not in $T$. This means that nodes in the remaining Clause-Gadget have to use the dummy sink $d_{0}$ to connect to the sink $r$. Thus, the node $s_{M}$ of the last Clause-Gadget $Q\left(C_{M}\right)$ is not in $T$ and neither are any of the padding nodes $d_{1}, d_{2}, \ldots, d_{L}$. Therefore, the size of $T$ is at most $J=n^{\epsilon}$, proving the theorem.

Observe this means that, from the perspective of the nodes, it is NP-hard to determine whether adding an extra node to its filtering list can lead to solutions where none of its packets ever route. In other words, it cannot avoid using an intermediate node it dislikes!

\section{Filtering: Anything-Goes!}

Here we consider the case where every node has an empty filtering list. This case is conceptually simple but still contains many technical difficulties involved in tracking packets when nodes become mistaken in their connectivity assessments. In this case, networks with no equilibrium can exist. Figure 2 presents such an example. Moreover, in this example, fair activation sequences exist where the node $v$ will never be in the sink component; for example, repeatedly activate nodes according to the permutation $\{v, u, w, x, y\}$. Despite this, every packet will route in two rounds! This example nicely illustrates the need to track packets if we want to understand the efficacy of BGP-like protocols.

For this class of preference functions, we show that the property of successful routing is universal. In any network, every packet will reach the sink. Specifically, we present a fair activation sequence of four rounds that routes every packet, even when there is no equilibrium.

Observe that when filtering lists are empty, a node $v$ only needs to known whether its neighbour $u$ has a path to the sink, as $v$ will never discount a path because it contains a node it dislikes. 


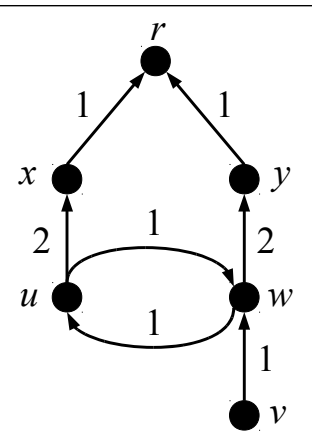

Figure 2: A network with no stable spanning tree. (Arc numbers indicate rankings, e.g., the number 2 on the arc $(u, x)$ means that $x$ is the second choice of $u$.)

Thus, we can view each node as having two states: clear or opaque. A node is clear if it is in the sink-component (the nomenclature derives from the fact that a packet at such a node will then reach the sink - that is, "clear"); otherwise, a node is opaque. Of course, as nodes update their chosen next-hop over time, they may be mistaken in their beliefs (inconsistent) as the routing graph changes. In other words, some clear nodes may not have "real" paths to the sink. After the learning step at the end of the round, these clear-opaque states become correct again.

Our algorithm and analysis are based on properties of the network formed by the first-choice arcs, called the first class network. We say that an arc $(u, v)$ of $G$ is a first-choice arc if $v$ is the most preferred neighbour of $u$. We denote the first class network by $F=\left(V, A_{1}\right)$, where $A_{1}$ is the set of the first-choice arcs. As in a routing graph $\mathcal{R}$, every node in $F$ has one outgoing arc. Thus, every component of $F$ is a 1-arborescence, a tree-like structure with either a cycle or a single node as a root. We denote the components of $F$ by $F_{0}, F_{1}, \ldots, F_{\ell}$, where $F_{0}$ is the component containing the sink $r$. Each $F_{j}$ has a unique cycle $C_{j}$, called a first class cycle. We may assume the first class cycle in $F_{0}$ is a self-loop at the sink $r$. Furthermore, when activated, every node in $F_{0}$ will always choose its neighbour in $F_{0}$; so, we may assume wlog that $F_{0}$ is the singleton node $\{r\}$. The routing graph at the beginning of round $t$ is denoted by $\mathcal{R}_{t}$. We denote by $\mathcal{K}_{t}$ and $\mathcal{O}_{t}$ the set of clear and the set of opaque nodes at the start of round $t$. Now we show that there is an activation sequence which routes every packet in four rounds.

The proof has two parts: a coordination phase and a routing phase. In the first phase, we give a coordination algorithm that generates a permutation that gives a red-blue colouring of the nodes with the following two properties:

(i) For each $F_{j}$, every node in $F_{j}$ has the same colour, i.e., the colouring is coordinated.

(ii) If the first class cycle $C_{j}$ of $F_{j}$ contains a clear node, then all nodes in $F_{j}$ must be coloured blue.

We remark that, subject to the these two properties, our algorithm will maximise the number of nodes coloured red, but this is not needed to prove that we can route a packet successfully.

The usefulness of this colouring mechanism lies in the fact that the corresponding permutation is a fair activation sequence that will force the red nodes to lie in the sink-component and the blue nodes to lie in non-sink components. Moreover, bizarrely, running this coordination algorithm four 
times in a row ensures that every packet routes! So, in the second phase (the routing phase), we simply run the coordination algorithm three more times.

\subsection{The Coordination Phase.}

The algorithm Coordinate $\left(\mathcal{K}_{t}\right)$ presented in Procedure 3 constructs a red-blue colouring of the nodes, i.e., a partition $(R, B)$ of $V$ (where $v \in R$ means that $v$ is coloured red and $v \in B$ means that $v$ is coloured blue) and which has the property that any node $v \in R$ prefers some node in $R$ to any node $w \in B$, and any node $v \in B$ prefers some node in $B$ to any node $w \in R$.

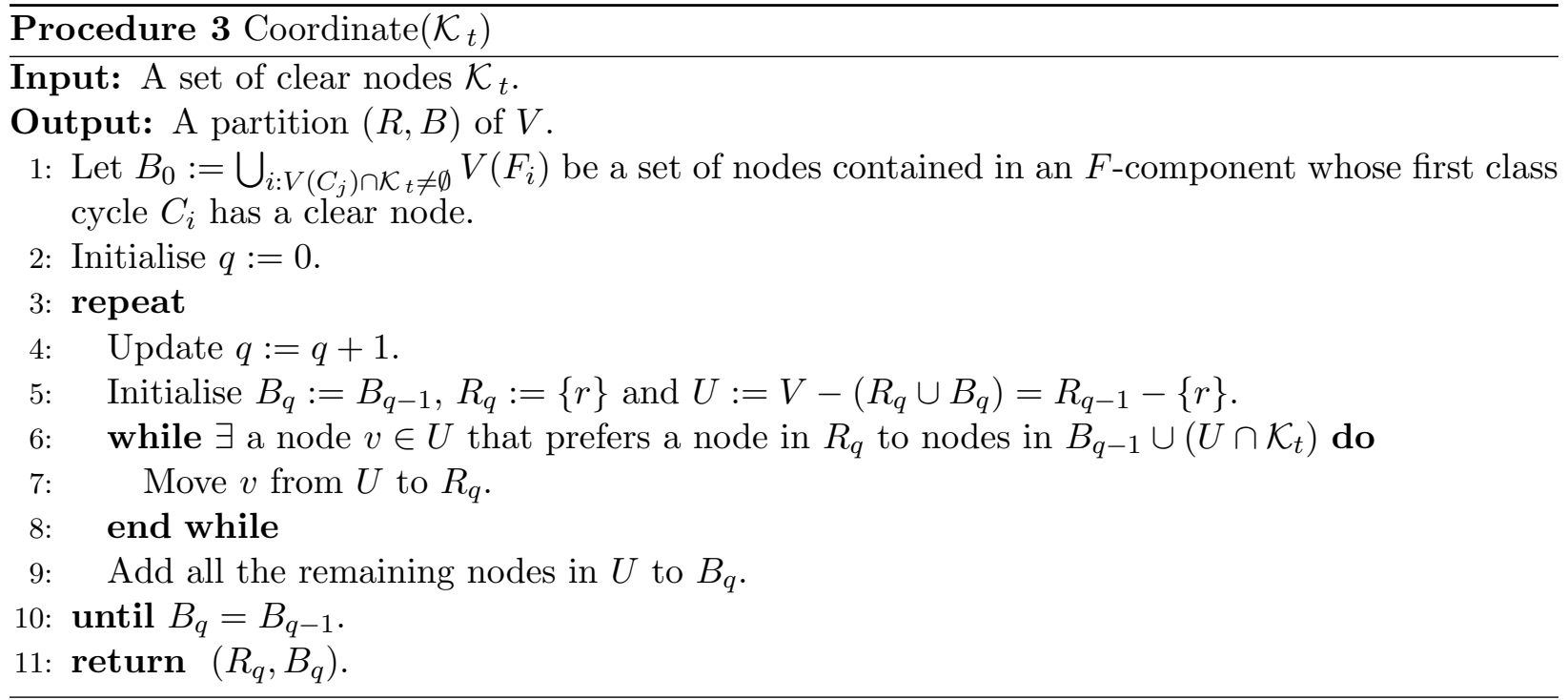

Observe that Coordinate $\left(\mathcal{K}_{t}\right)$ contains many loops. However, we only wish to generate a single activation sequence $\pi_{t}$ from the procedure. To do this, we take the output partition $(R, B)$ and use it to build an activation sequence.

We begin by activating nodes in $B_{0}=\bigcup_{i: C_{i} \cap \mathcal{K}_{i} \neq \emptyset} V\left(F_{i}\right)$, the components $F_{i}$ whose first class cycles contain at least one clear node. To order the nodes of $B_{0}$, we proceed as follows. For each $F_{i}$ with $V\left(F_{i}\right) \subseteq B_{0}$, take a clear node $v \in C_{i} \cap \mathcal{K}_{t}$. Then activate the nodes of $F_{i}$ (except $v$ ) in increasing order of distance from $v$ in $F_{i}$, and after that activate $v$. This forms a non-sinkcomponent $F_{i}$ in the routing graph as every node can choose its first-choice. Next, we activate the remaining nodes in $B$. We order the nodes of $B-B_{0}$ in a greedy fashion; a node can be activated once its most-preferred clear neighbour is in $B$. Finally, we activate the nodes in $R$. Again, this can be done greedily. Specifically, we activate nodes of $R$ in the same order as when they were added to $R$.

Lemma 6. Given a partition $(R, B)$ from Coordinate $\left(\mathcal{K}_{t}\right)$, the activation sequence $\pi_{t}$ induces a sink-component on $R$ and non-sink-components on $B$.

Proof. First, let us verify by induction that each node in $R$ ends up in the sink-component. For the base case, the sink $r$ is clearly in the sink-component. Now, the red nodes are activated after the blue nodes. Let $q^{*}$ be the last iteration of Coordinate $\left(\mathcal{K}_{t}\right)$; hence, $B_{q^{*}}=B_{q^{*}-1}$. So, at the start of this iteration, $B_{q^{*}}=B$ and $R_{q^{*}}=\{r\}$. Despite this, at the end of the round, we have $R_{q^{*}}=R$. This implies that every node $v$ of $R$ prefers a node of $R_{q^{*}}$ to a node of $B_{q^{*}-1} \cup\left(U \cap \mathcal{K}_{t}\right)=B \cup\left(U \cap \mathcal{K}_{t}\right) \supseteq B$ 
when it is added to $R_{q^{*}}$. But, then $\pi_{t}$ orders the nodes such that, on activation, every node $v$ of $R$ prefers a node of $R_{q^{*}}$ (which are in the sink component by induction) to a node of $B$. Thus, regardless of which components the blue nodes were placed in upon activation, all the red nodes are placed in the sink-component (it can only help if the some of the red nodes were placed in the sink-component).

Now, let us show that the blue nodes do not end up in the sink-component. By the construction of $\pi_{t}$, the nodes of $B_{0}$ connect together via their first class components. Consequently, they belong to non-sink components. Finally, consider the nodes in $B-B_{0}$. Observe that the size of $B_{q}$ increases in each iteration, that is, $B_{q} \subsetneq B_{q-1}$ for all $q<q^{*}$. So, at the end of their iterations, $R_{q} \subseteq R_{q-1}$. Now, take a node $v$ added to $B_{q}$ in Step 9 in iteration $q$. At this point, the remaining nodes are $U=R_{q-1}-R_{q}$, and $v$ prefers some node $w$ in $B_{q-1} \cup\left(U \cap \mathcal{K}_{t}\right)$ to any node in $R_{q}$ (or has no neighbours in $R_{q}$ at all $)$. But, $B_{q-1} \cup\left(U \cap \mathcal{K}_{t}\right)=B_{q-1} \cup\left(R_{q-1}-R_{q} \cap \mathcal{K}_{t}\right) \subseteq B_{q-1} \cup\left(R_{q-1}-R_{q}\right)=B_{q}$. Thus, $v$ either prefers some node in $B_{q^{*}}$ to any node in $R_{q^{*}}$ or has no neighbours in $R_{q^{*}}$. But, by assumption, every node has a path to the sink in the "all-choice" graph $G$. So, when considering the blue nodes, there must be a node in $B-B_{0}$ that has its most preferred "clear" neighbour in $B$. Therefore, $\pi_{t}$ induces non-sink components on $B$.

The coordination and other desirable properties hold when we apply $\pi_{t}$.

Lemma 7. Given the activation sequence $\pi_{t}$ for $(R, B)$, at the end of the round, the following hold:

- The sink-component includes $R$ and excludes $B$.

- Coordination: For each $F_{i}$, either all the nodes of $F_{i}$ are in the sink-component or none of them are.

- Let $B_{0}=\bigcup_{i: V\left(C_{i}\right) \cap \mathcal{K}_{t} \neq \emptyset} V\left(F_{i}\right)$ and suppose $\mathcal{K}_{t}=B_{0}$. If a packet travels for $n$ hops but does not reach the sink, then it must be at a node in $\mathcal{K}_{t}$.

Proof. The first statement follows Lemma 6.

For the second statement, it suffices to show that, for each $F_{i}$, either $V\left(F_{i}\right) \subseteq R$ or $V\left(F_{i}\right) \subseteq B$. If not, then there is an $F_{i}$ containing both red and blue nodes. Thus, there are two possibilities.

(i) There is a node $v \in F_{i}$ such that $v$ is in $R$ and its first choice is $w \in B$. This is not possible. To see this, observe that we must have $w \in B_{q-1}$ because of the stopping condition $B_{q}=B_{q-1}$. But, then $v$ could not be added to $R_{q}$, a contradiction. (ii) There is a node $v \in F_{i}$ such that $v$ is in $B$ and its first choice is $w \in R$. Again, this is not possible. To see this, observe that we must have $v \notin B_{0}$ because each $B_{0}$ consists only of first class components that are monochromatic blue. Recall also that $R_{q} \subseteq R_{q-1}$ for all $q$. Thus, we must have $w \in R_{q}$ for all $q$. But, then $v$ would have been added to $R_{q}$, a contradiction. For the third statement, note that a packet that travels for $n$ hops but does not reach the sink must be stuck in some cycle. Consider the construction of $(R, B)$. Since $\mathcal{K}_{t}=B_{0}$, we only add a node to $B$ whenever it prefers some node in $B$ to any node in $R$. Because $U \cap \mathcal{K}_{t}=\emptyset$, nodes in $B-B_{0}$ cannot form a cycle on their own. Thus, the packet is stuck in a cycle that contains a clear node; the only such cycles are the first class cycles of $B_{0}$ since $\mathcal{K}_{t}=B_{0}$.

The following lemma follows by the construction of a partition $(R, B)$.

Lemma 8. Let $\left(R^{\prime}, B^{\prime}\right)$ be any partition generated from the procedure Coordinate(), and let $(R, B)$ be a partition obtained by calling Coordinate $\left(\mathcal{K}_{t}\right)$ where $\bigcup_{i: V\left(C_{i}\right) \cap \mathcal{K}_{t} \neq \emptyset} V\left(F_{i}\right) \subseteq B^{\prime}$. Then $R^{\prime} \subseteq R$. 
Proof. Consider a partition $\left(R_{q}, B_{q}\right)$ constructed during a call to Coordinate $\left(\mathcal{K}_{t}\right)$. Observe that $B_{0} \subseteq B^{\prime}$ because $B_{0}=\bigcup_{i: V\left(C_{i}\right) \cap \mathcal{K}_{t} \neq \emptyset} V\left(F_{i}\right)$. By the construction of $\left(R^{\prime}, B^{\prime}\right)$, since $B_{0} \subseteq B^{\prime}$, every node of $R^{\prime}$ must have been added to $R_{1}$, i.e., $R^{\prime} \subseteq R_{1}$. Inductively, if $R^{\prime} \subseteq R_{q}$ for some $q \geq 1$, then $B_{q} \subseteq B^{\prime}$ and thus $R^{\prime} \subseteq R_{q+1}$ by the same argument.

\subsection{The Routing Phase}

Running the coordination algorithm four times ensures every packet will have been in the sinkcomponent at least once, and thus, every packet routes.

Theorem 9. In four rounds, every packet routes.

Proof. The first round $t=1$ is simply the coordination phase. We will use subscripts on $R$ and $B$ (e.g., $R_{t}$ and $B_{t}$ ) to denote the final colourings output in each round and not the intermediate sets $R_{q}$ and $B_{q}$ used in Coordinate(). Now, consider a packet generated by any node of $V$. First, we run Coordinate $\left(\mathcal{K}_{1}\right)$ and obtain a partition $\left(R_{1}, B_{1}\right)$. By Lemma 7 , if the packet is in $R_{1}$, then it is routed successfully, and we are done. Hence, we may assume that the packet does not reach the sink and thus must be in $B_{1}$. Note that, now, each $F_{i}$ is either contained in $R_{1}$ or $B_{1}$ by Lemma 7 .

We now run Coordinate $\left(\mathcal{K}_{2}\right)$ and obtain a partition $\left(R_{2}, B_{2}\right)$. By Lemma $7, \mathcal{K}_{2}=R_{1}$. So, if the packet does not reach the sink, it must be in $B_{2}$. Since no first class component intersects both

$R_{1}$ and $B_{1}$, we have $R_{1}=\mathcal{K}_{2}=\bigcup_{i: V\left(C_{i}\right) \cap \mathcal{K}_{2} \neq \emptyset} V\left(F_{i}\right)$. Thus the nodes $\mathcal{K}_{2}$ are all initially coloured blue in Step 1 of Coordinate $\left(\mathcal{K}_{2}\right)$. As the set of blue nodes only expands throughout the round

So, $R_{1} \subseteq B_{2}$ (since $\mathcal{K}_{2} \subseteq B_{2}$ ) and $R_{2} \subseteq B_{1}$, and Lemma 7 implies that the packet is in $R_{1}$.

Third, we run Coordinate $\left(\mathcal{K}_{3}\right)$ and obtain a partition $\left(R_{3}, B_{3}\right)$. Applying the same argument as before, we have that the packet is in $R_{2}$ (or it is routed), $R_{2} \subseteq B_{3}$ and $R_{3} \subseteq B_{2}$. Now, we run Coordinate $\left(\mathcal{K}_{4}\right)$ and obtain a partition $\left(R_{4}, B_{4}\right)$. By Lemma 7, we have $\mathcal{K}_{4}=\bigcup_{i: V\left(C_{i}\right) \cap \mathcal{K}_{4} \neq \emptyset} V\left(F_{i}\right)$. Since $R_{3}=\mathcal{K}_{4} \subseteq B_{2}$, Lemma 8 implies that $R_{2} \subseteq R_{4}$. Thus, the packet is routed successfully since $R_{4}$ is contained in the sink-component.

\section{Filtering: Not-Me!}

In practice, it is important to try to prevent cycles forming in the routing graph of a network. To achieve this, loop-detection is implemented in the BGP-4 protocol [17]. The "Not-Me!" filtering encodes loop-detection in the BGP-4 protocol simply by having a filtering list $\mathcal{D}(v)=\{v\}$, for every node $v$. For this class of preference function, we again show that every packet will route. Recall, this is in contrast to Theorem 5, which says that it is NP-hard to determine whether we can route every packet for general filtering lists of cardinality one. Moreover, we exhibit a constructive way to obtain a stable spanning tree via fair activation sequences. Interestingly, all of the packets will have routed before stability is obtained. In particular, we give an algorithm that constructs an activation sequence such that every packet routes successfully in $\frac{1}{3} n$ rounds whereas the network itself becomes stable in $n$ rounds.

This result is the most complicated part of our paper, so we will first give a high level overview. Clearly, when filtering lists are non-empty, we have an additional difficulty: even if $w$ is the most preferred choice of $v$ and $w$ has a non-empty routing path $P$, $v$ still may not be able to choose $w$ because $P$ contains a node on $v$ 's filter list (in this case, $v$ itself). This can cause the routing graph to evolve in ways that are very difficult to keep track of. Thus, the key idea is to design activation 
permutations that manipulate the routing graph in a precise and minor fashion in each round. To do this, we search for a spanning tree with a Strong Stability Property.

Property 10 (Strong Stability - Informal). A spanning tree $S$ has the strong stability property on $\mathbb{O} \subseteq V$ if and only if, for every node $v \in \mathbb{O}$, the most preferred choice of $v$ is its parent $w$ in $S$, even if $v$ can choose any node outside $\mathbb{O}$ and any node outside its subtree in the forest $S[\mathbb{O}]$.

To illustrate this property, consider a simple setting where $S$ is just the path $S=(e, d, c, b, a, r)$ and $\mathbb{O}=\{b, c, e\}$. If $S$ is strongly stable on $\mathbb{O}$, then $b$ must prefer $a$ to nodes in $\{r, d, e\}$. Observe that $e$, whilst a descendent of $b$ in $S$, is not a descendent of $b$ in $S[\{b, c, e\}]$. So, even if $b$ is allowed to choose $e$, which is a descendent of $b$ in $S, b$ still wants to choose $a$ as its parent.

Thus, the strong stability property says that the choice of a vertex $v \in \mathbb{O}$ in $S$ is the best one even if all the nodes outsides $\mathbb{O}$ change their choices. (that is, even if we replace $S[V-\mathbb{O}]$ with a completely different subgraph). For the special case where $\mathbb{O}=\mathcal{O}$, the set of opaque nodes, if we activate nodes of $S$ in increasing order of distance from the sink $r$ then every node in $\mathcal{O}$ will choose its parent in $S$ - as the clear nodes in $S_{v}$ are not desirable to connect to. As we will see, under certain conditions, we can even maintain the choices of nodes in $\mathbb{O}$ even if some of them are clear and some are opaque.

A stable spanning tree, a tree where no node wants to change its choice, can be found in polynomial-time, and given a stable spanning tree $S$, it is easy to force opaque nodes in $\mathcal{O}_{t}$ to make the same choices as in $S$. But, this only applies to the set of opaque nodes, which changes with each round. The strong stability property allows us to make a stronger manipulation. Intuitively, the strong stability property says that once we force every node $v \in \mathbb{O}$ to make the same choice as in $S$, we can maintain these choices in all the later rounds. Moreover, in each round, if we cannot route all the packets, then we can make the strong stability property spans three more nodes; otherwise, the property spans one more node. Thus, in $\frac{1}{3} n$ rounds, every packet will route, but we need $n$ rounds to obtain stability.

With this overview complete we introduce some formal definitions needed for the proof. Again, $\mathcal{O}_{t}$ and $\mathcal{K}_{t}$ denote the set of opaque and clear nodes, respectively, at the beginning of round $t$. Given a graph $R$ and a set of nodes $U$, we denote by $R[U]=\{(u, v): u, v \in U,(u, v) \in R\}$ the subgraph of $R$ induced by $U$, and we denote by $\mathcal{A}_{R}^{+}(U)=\{(u, v): u \in U,(u, v) \in R\}$, the subgraph of arcs of $R$ induced by $U$ plus arcs leaving $U$.

Given a set of nodes $Q \subseteq V$, the $Q$-subtree of $v$, with respect to a tree $T$, is the maximal subtree rooted at $v$ of the forest $T[Q]$. A (spanning) tree $T$ is stable if every node $v$ with $(v, w) \in T$, prefers its parent $w$ in $T$ to every non-descendant; thus, no node wants to change its next hop. A spanning tree $S$ has the strong stability property on the set of nodes $\mathbb{O}$ if every node $v \in \mathbb{O}$ with $(v, w) \in S$ prefers its parent $w$ to every node outside its $\mathbb{O}$-subtree; observe that if $\mathbb{O}=V$ (or $V-\{r\}$ ), then $S$ is also stable. We say that $S$ is a skeleton of a (non-spanning) tree $T$ if, for every (maximal) subtree $F \subseteq S[\mathbb{O}]$, either $T$ contains $\mathcal{A}_{S}^{+}(F)$ or $T$ contains no node of $F$.

\subsection{Finding a Strongly Stable Tree}

In this section, we present a subroutine for finding a spanning tree with the strong stability property. The input of this algorithm (see Procedure 4) is a sink-component $T^{i n}$ and a spanning tree $S^{\text {in }}$ with the strong stability property on a given set of nodes $\mathbb{O}$. The algorithm expands the strong stability property to also hold on $\mathcal{O}$, the set of nodes not in the sink-component $T^{i n}$. 
Procedure 4 FindStable $\left(T^{\text {in }}, S^{\text {in }}, \mathbb{O}\right)$

Input: A sink-component $T^{\text {in }}$ and a spanning (or empty) tree $S^{\text {in }}$ such that

(1) The tree $S^{i n}$ has the strong stability property on $\mathbb{O}$, and

(2) $S^{i n}$ is a skeleton of $T^{\text {in }}$.

Output: A stable spanning tree $S^{\text {out }}$ with the strong stability property on $\mathbb{O} \cup \mathcal{O}$, where $\mathcal{O}=$ $V-V\left(T^{i n}\right)$.

1: Let $\mathcal{O}=V-V\left(T^{i n}\right)$ be the set of nodes not in the sink component $T^{i n}$.

2: Initialise $S^{\text {out }}:=T^{\text {in }} \cup \mathcal{A}_{S^{i n}}^{+}(\mathcal{O})$.

3: Initialise $\mathcal{C}_{1}:=S^{\text {out }}[\mathcal{O}]$.

4: for iteration $t:=1$ to $|\mathcal{O}|$ do

5: $\quad$ Pick an arbitrary leaf $v$ of $\mathcal{C}_{t}$.

6: $\quad$ Pick a node $w \in V\left(S^{\text {out }}\right)$ such that $v$ prefers $w$ to any other node not in its $\mathcal{O}$-subtree in $S^{\text {out }}$.

7: $\quad$ Replace the $\operatorname{arc}(v, y)$ in $S^{\text {out }}$ by the $\operatorname{arc}(v, w)$.

8: $\quad$ Update $\mathcal{C}_{t+1}:=\mathcal{C}_{t}-\{v\}$.

9: end for

10: return $S^{\text {out }}$.

Before proving the correctness of the procedure FindStable $\left(T^{i n}, S^{i n}, \mathbb{O}\right)$, we prove some basic facts.

Lemma 11 (Union Lemma). Let $S$ be a spanning tree that is strongly stable on $A \subseteq V$ and also on $B \subseteq V$. Then $S$ is strongly stable on $Q=A \cup B$.

Proof. Without loss of generality, take a vertex $v \in A \subseteq Q$. Let $F_{A}$ and $F_{Q}$ be the (maximal) $A$-subtree and $Q$-subtree of $v$ in $S$, respectively. Then $V\left(F_{A}\right) \subseteq V\left(F_{Q}\right)$ because $A \subseteq Q$. By the strong stability property of $S$ on $A$, we have that $v$ prefers its parent $w$ in $S$ to any other node in $V-V\left(F_{A}\right)$. But, $V-V\left(F_{Q}\right) \subseteq V-V\left(F_{A}\right)$. It follows that $S$ is strongly stable on $Q=A \cup B$.

The next lemma proves an important property of a skeleton of a tree $T$.

Lemma 12 (Skeleton Lemma). Let $T$ be any tree. Let $S$ be a spanning tree that is strongly stable on a set of vertices $\mathbb{O}$ and be a skeleton of $T$. Let $\mathcal{O}=V-V(T)$. Then, for any spanning tree $T^{\prime}$ such that $T \subseteq T^{\prime}$, the tree $T^{\prime}$ is strongly stable on $\mathbb{O}-\mathcal{O}$.

Proof. Consider any node $v \in \mathbb{O}-\mathcal{O}$. Let $F_{v}$ be the $\mathbb{O}$-subtree of $v$ in $S$. By the definition of skeleton, for any (maximal) subtree $F \subseteq S[\mathbb{O}]$, either (i) $\mathcal{A}_{S}^{+}(F) \subseteq T$ or (ii) $V(F) \cap V(T)=\emptyset$. Since $v \in V(T) \cap \mathbb{O}$, it must be that $\mathcal{A}_{S}^{+}\left(F_{v}\right) \subseteq T \subseteq T^{\prime}$. Thus, $V\left(F_{v}\right) \subseteq \mathbb{O}-\mathcal{O}$ as $V(T)=V-\mathcal{O}$. Therefore $F_{v}$ is also the $(\mathbb{O}-\mathcal{O})$-subtree of $v$ in $S$. By the strong stability property of $S$ on $\mathbb{O}$, we know that $v$ prefers its parent $w$ in $S$ to any node in $V-V\left(F_{v}\right)$. So, $S$ has the strong stability property on $\mathbb{O}-\mathcal{O}$.

The next lemma shows the correctness of the procedure $\operatorname{Stabilise}\left(T^{i n}, S^{i n}, \mathbb{O}\right)$.

Lemma 13. The procedure FindStable $\left(T^{i n}, S^{\text {in }}, \mathbb{O}\right)$ outputs a spanning tree $S^{\text {out }}$ with the strong stability property on $\mathbb{O} \cup \mathcal{O}$. 
Proof. To begin, we show that $S^{\text {out }}$ is a spanning tree throughout the procedure. Initially $S^{\text {out }}=$ $T^{\text {in }} \cup \mathcal{A}_{S^{i n}}^{+}(\mathcal{O})$. Therefore, $S^{\text {out }}$ contains every node, since $\mathcal{O}=V-V\left(T^{i n}\right)$. Let us see that $S^{\text {out }}$ is also connected. As $S^{i n}$ is a spanning tree, we have that each component $F$ of $\mathcal{A}_{S^{i n}}^{+}(\mathcal{O})$ is a tree (arborescence). Furthermore as the sink $r$ is not in $\mathcal{O}$, there is exactly one arc in $\mathcal{A}_{S^{i n}}^{+}(\mathcal{O})$ leaving $F$ (from its root) and entering $V\left(T^{i n}\right)$. Thus, $S^{\text {out }}=T^{i n} \cup \mathcal{A}_{S^{i n}}^{+}(\mathcal{O})$ is a spanning tree.

Now, consider how $S^{\text {out }}$ changes during the loop phase of the procedure. No node in $V-\mathcal{O}$ is considered during this phase, so $S^{\text {out }}$ never contains any arc leaving $V-\mathcal{O}$ and entering $\mathcal{O}$. As a result, the $\mathcal{O}$-subtree of $v$ in $S^{\text {out }}$ coincides exactly with the set of all descendants of $v$ in $S^{\text {out }}$. Hence, in Step 6, node $v$ never selects a descendant node to be $w$. So, we can safely replace the arc $(v, y) \in S^{\text {out }}$ by the arc $(v, w)$ without creating a cycle. This shows that $S^{\text {out }}$ is always a spanning tree.

Next, we show that $S^{\text {out }}$ has the strong stability property both on $\mathbb{O}-\mathcal{O}$ and on $\mathcal{O}$. By Lemma 11, this will imply that $S^{\text {out }}$ is strongly stable on $\mathbb{O} \cup \mathcal{O}$. Now, $S^{\text {in }}$ is strongly stable on $\left(\mathbb{O}\right.$ and is a skeleton on $T^{\text {in }}$. Furthermore, $T^{\text {in }} \subseteq S^{\text {out }}$ by construction. Thus, applying Lemma 12 , we have that $S^{\text {out }}$ is strongly stable on $\mathbb{O}-\mathcal{O}$. It only remains to show that $S^{\text {out }}$ is strongly stable on $\mathcal{O}$. To achieve this, we show by induction that $S^{\text {out }}$ is strongly stable on $\mathcal{L}_{t}=\mathcal{O}-V\left(C_{t}\right)$ in each iteration $t$. (Note that, on termination, $\mathcal{L}_{t}=\mathcal{O}$.) This is true for $t=1$ as $\mathcal{L}_{1}=\emptyset$. Now, consider iteration $t>1$, and assume that strong stability holds on $\mathcal{L}_{t-1}$. Observe that no node $u \in \mathcal{O}-\mathcal{L}_{t-1}$ has a parent $x \in \mathcal{L}_{t-1}$; otherwise, $x$ would have not been added to $\mathcal{L}_{t-1}$. Since $v$ is

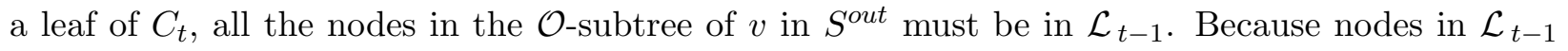
can not change their parents after this time, every descendant of $v$ in $\mathcal{O}$ will remain a descendant of $v$. Consequently, $v$ prefers $w$ to other any non-descendant in $S^{o u t}$ throughout the rest of the procedure. Thus, $S^{\text {out }}$ is strongly stable on $\mathcal{L}_{t}$.

\subsection{Routing Every Packet in $n$ Rounds.}

We are now ready to present an algorithm that routes every packet in $n$ rounds (recall that each round consists of a single fair-activation sequence). In addition to the procedure FindStable(), two procedures (namely, Procedures 5 and 6) based upon a breath-first-search (BFS) algorithm are our basic building block for generating an activation sequence. Given a spanning tree $F$ and a set of nodes $U \subseteq V$, the procedure $\operatorname{BFS}(U, F)$ activates the nodes of $U$ in breadth-first-search (BFS) order. That is, the nodes of $U$ are activated in increasing order of distance to the sink $r$ in $F$.

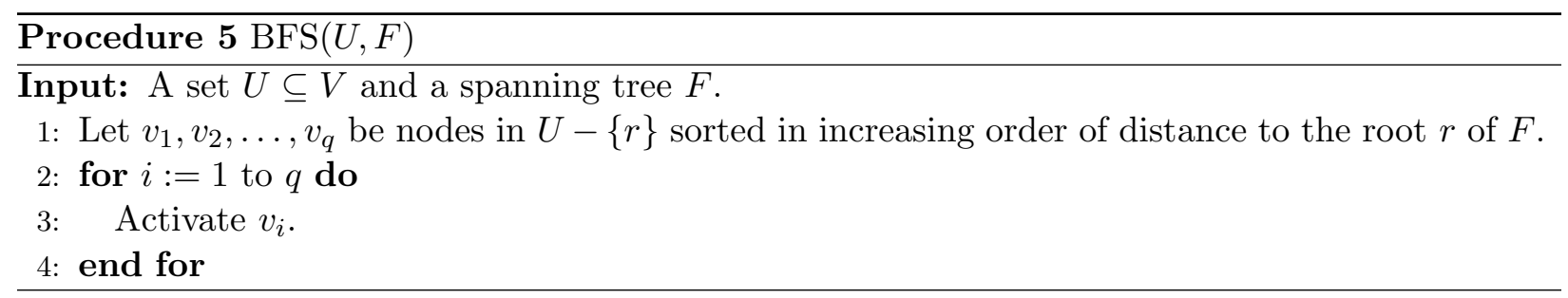

Similarly, the procedure reverse-BFS $(U, F)$ activates the nodes of $U$ in breadth-first-search (BFS) reverse-order.

Over the course of these $n$ rounds, the main algorithm (Procedure 7) utilises these three procedures on the following two classes of nodes.

(1) The set of nodes that have been clear in every round up to time $t$, denoted by $\mathbb{K}_{t}=\cap_{i=1}^{t} \mathcal{K}_{i}$. 


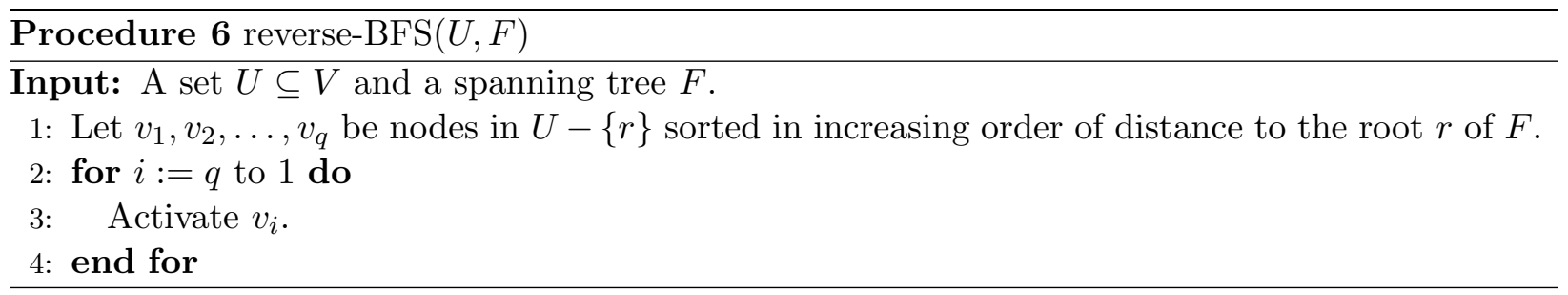

(2) The complement of $\mathbb{K}_{t}$, which is the set of nodes that have been opaque at least once by time $t$, denoted by $\mathbb{O}_{t}=\cup_{i=1}^{t} \mathcal{O}_{i}$.

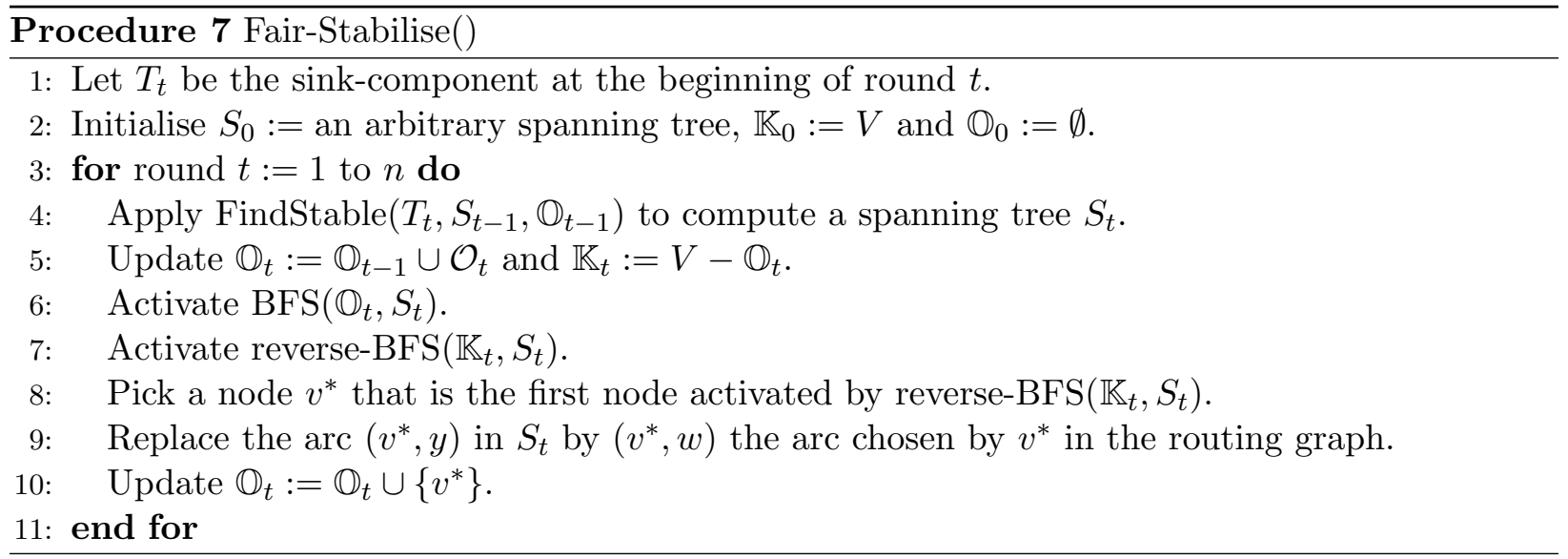

Observe that Procedure 7 is clearly fair because $\mathbb{K}_{t}$ and $\mathbb{O}_{t}$ partition the set of nodes. The basic intuition behind the method is that if we can make our routing graph $T_{t+1}$ look like the spanning tree $S_{t}$, then every packet will route. Typically, any activation sequence that attempts to do this, though, will induce inconsistencies. This, in turn, will force nodes to go opaque. But, it turns out that we can make those nodes in $\mathbb{O}_{t}$ choose arcs in accordance with $S_{t}$ via the use of BFS $\left(\mathbb{O}_{t}, S_{t}\right)$. (It is not at all obvious that this can be done because nodes in $\mathbb{O}_{t}$ may actually be clear, that is, they need not be in $\mathcal{O}_{t}$.)

Then the question becomes how do we keep track of the packets. The key point is that nodes in $\mathbb{O}_{t}$ choose arcs in accordance with the spanning tree $S_{t}$. Therefore, since $\mathbb{O}_{1} \subset \mathbb{O}_{2} \subset \mathbb{O}_{3} \subset \ldots$ and the containments are strict, we will eventually have $\mathbb{O}_{t}=V$, and our routing graph will be a spanning tree. Thus, every packet routes! Moreover, the strong stability property of $S_{t}$ on $\mathbb{O}_{t}=V$ also implies that the final routing graph is a stable spanning tree.

The following lemma presents the key properties we need to prove all this.

Lemma 14. At the end of round $t$ of Fair-Stabilise(), we have that:

- The spanning tree $S_{t}$ has the strong stability on $\mathbb{O}_{t}$, and

- $\mathcal{A}_{S_{t}}^{+}\left(\mathbb{O}_{t}\right)$ is contained in the routing graph at the end of round. Specifically, for each node $v \in \mathbb{O}_{t}$, if $(v, w) \in S_{t}$ then, upon activation, $v$ chooses $w$ as its next hop.

Proof. We proceed by induction on $t$. The statement is clearly true for $t=0$ because $\mathbb{O}_{0}=\emptyset$. Now, suppose that the statements hold up to round $t-1$ for some $t>0$. We first show that $S_{t}$ has a strong stability property on $\mathbb{O}_{t}$ (before adding $v^{*}$ ). To do this, we have to show that 
FindStable $\left(T_{t}, S_{t-1}, \mathbb{O}_{t-1}\right)$ is called with a valid input, i.e., $S_{t-1}$ is a skeleton of $T_{t}$ and has the strong stability property on $\mathbb{O}_{t-1}$. The latter fact, that $S_{t-1}$ has the strong stability property on $\mathbb{O}_{t-1}$, follows from the induction hypothesis.

So, we need to show $S_{t-1}$ is a skeleton of $T_{t}$. By induction, at the end of the previous round (before the learning phase) we have that $\mathcal{A}_{S_{t-1}}^{+}\left(\mathbb{O}_{t-1}\right)$ is contained in the routing graph. So, for each (maximal) subtree $F \subseteq S_{t-1}\left[\mathbb{O}_{t-1}\right]$ rooted at a node $v$, every node of $F$ has a path to the sink $r$ if and only if $v$ has a path to $r$. Thus, either $\mathcal{A}_{S_{t-1}}^{+}(F)$ is contained in the sink component $T_{t}$ or no node of $F$ is in the sink component $T_{t}$. In other words, $S_{t-1}$ is a skeleton of $T_{t}$, as desired. Consequently, by Lemma 13 , FindStable $\left(T_{t}, S_{t-1}, \mathbb{O}_{t-1}\right)$ builds a spanning tree $S_{t}$ that is strongly stable on $\mathbb{O}_{t}$ (before the arc emanating from $v^{*}$ is updated in Step 8).

Next, we claim that after applying $\operatorname{BFS}\left(\mathbb{O}_{t}, S_{t}\right)$, the routing graph looks exactly like $S_{t}$. This is true for all nodes outside $\mathbb{O}_{t}$ by the construction of $S_{t}$. To see this, observe that the tree $S_{t}$ is constructed from FindStable $\left(T_{t}, S_{t-1}, \mathbb{O}_{t-1}\right)$ by adding arcs joining opaque nodes in $\mathcal{O}_{t} \subseteq \mathbb{O}_{t}$ to $V\left(T_{t}\right)$. Since no nodes in $V-\mathbb{O}_{t}$ have been activated at this point, we must have that $T_{t}\left[V-\mathbb{O}_{t}\right] \subseteq$ $T_{t}\left[V-\mathcal{O}_{t}\right]=S_{t}\left[V-\mathcal{O}_{t}\right]$. For the remaining nodes, we proceed by induction on the order in which nodes are activated by $\operatorname{BFS}\left(\mathbb{O}_{t}, S_{t}\right)$. Our induction hypothesis is that every node activated during this time will choose its parent in $S_{t}$ as its next hop and become consistent, so it has a "real" path as its chosen route.

Let us prove the base case. Consider the first node $x$ activated by $\operatorname{BFS}\left(\mathbb{O}_{t}, S_{t}\right)$. Suppose $x$ chooses $z$ in the routing graph, that is, $(x, z) \in T_{t}$. At the time we activate $v^{*}$, we have (1) $T_{t}\left[V-\mathcal{O}_{t}\right]=S_{t}\left[V-\mathcal{O}_{t}\right]$ and $(2) z$ is clear and is in $V-\mathbb{O}_{t}$ (because the sink node $r$ is in $V-\mathbb{O}_{t}$ ). There are two cases. First, if $x$ is opaque, then $z$ is valid for $x$ because every node now has a real path as its chosen route. Second, if $x$ is clear, then $z$ cannot have $x$ in its chosen route because $(x, z)$ is in the routing graph. Also, each node in the $\mathbb{O}_{t}$-subtree of $x$ in $S_{t}$ either is a descendant of $x$ or is opaque by (1). Thus, by the strong stability property of $S_{t}$ on $\mathcal{O}_{t}, z$ is the best valid choice of $x$. Consequently, $x$ must choose $z$ and must be consistent upon activation because $z$ has a real path as its chosen route.

Inductively, assume that every node activated before $x$ by BFS $\left(\mathbb{O}_{t}, S_{t}\right)$ chooses its parent in $S_{t}$ as its next hop and is consistent at the time we activate $x$. Now activate $x$ and let $Q_{0}$ be the set of nodes already activated by $\operatorname{BFS}\left(\mathbb{O}_{t}, S_{t}\right)$. Then $R[Q]=S_{t}[Q]$ where $R$ is the current routing graph and $Q=(V-\mathcal{O}) \cup Q_{0}$. Thus, by induction, the node $x$ must have a parent $z \in Q$ which is clear and consistent. But this situation is now similar to case where we activated the first node (with $Q$ replacing $V-\mathbb{O}_{t}$ ). Therefore, we conclude that $x$ must choose $z$ and become consistent. This proves the claim that the routing graph looks like $S_{t}$.

Finally, consider the node $v^{*}$. By the choice of $v^{*}$, all the descendants of $v^{*}$ in $S_{t}$ must be in $\mathbb{O}_{t}$. Since the routing graph now looks exactly like $S_{t}$ and every node is consistent, $v^{*}$ can choose any node outside its $\mathbb{O}_{t}$-subtree in $S_{t}$. Consequently, as $w$ is the best valid choice of $v^{*}$, the modification of the tree $S_{t}$ (replacing $\left(v^{*}, y\right)$ by $\left(v^{*}, w\right)$ ) results in a tree $S_{t}$ that is strongly stable on $\mathbb{O}_{t}$ after adding $v^{*}$. Also, since $\left(v^{*}, w\right)$ is now in both the routing graph and $S_{t}$, both statements hold at the end of the iteration.

Lemma 14 produces a guarantee that the cardinality of $\mathbb{O}_{t}$ is increasing.

Lemma 15. The cardinality of $\mathbb{O}_{t}$ strictly increases with each round $t$, until $\mathbb{O}_{t}=V$. Furthermore if some packet does not route in round $t-1$, then $\left|\mathbb{O}_{t}\right| \geq\left|\mathbb{O}_{t-1}\right|+3$. 
Proof. By construction, we have $\left|\mathbb{O}_{t}\right| \geq\left|\mathbb{O}_{t}\right|+1$. Thus, the first statement holds. To prove the second statement, it suffices to show that if there is an opaque node in $\mathcal{O}_{t}$ then there are at least two opaque nodes not in $\mathbb{O}_{t-1}$. This would imply that we add at least three nodes to $\mathbb{O}_{t}$, two nodes from $\mathcal{O}_{t}-\mathbb{O}_{t-1}$ and the node $v^{*}$ at the end of the iteration. So, we have to prove that $\left|\mathcal{O}_{t}-\mathbb{O}_{t-1}\right| \geq 2$ for all $t \geq 1$.

Suppose $\mathcal{O}_{t} \neq \emptyset$; if not then every packet routed in round $t-1$. Now consider the routing graph $R_{t-1}$ at the end of round $t-1$ (before the learning phase). Since there is an opaque node in $\mathcal{O}_{t}$, the routing graph $R_{t-1}$ must contain a cycle $C$. By Lemma $14, \mathcal{A}_{S_{t-1}}^{+}\left(\mathbb{O}_{t-1}\right)$ is contained in $R_{t-1}$. The subgraph $\mathcal{A}_{S_{t-1}}^{+}\left(\mathbb{O}_{t-1}\right)$ is a forest because $\mathcal{A}_{R_{t-1}}^{+}\left[\mathbb{O}_{t-1}\right]=\mathcal{A}_{S_{t-1}}^{+}\left[\mathbb{O}_{t-1}\right]$ and $S_{t-1}$ is a tree. So, at least one vertex of $C$ is not in $\mathbb{O}_{t-1}$. Thus, we have shown that $\left|\mathcal{O}_{t}-\mathbb{O}_{t-1}\right| \geq 1$. If $C$ has no vertex in $\mathbb{O}_{t-1}$, then $C$ must have at least two vertices and all of them are in $\mathcal{O}_{t}-\mathbb{O}_{t-1}$, so we are done. If $C$ has some vertex in $\mathbb{O}_{t-1}$, then $C$ must contain an arc $(u, v)$ such that $u \in \mathbb{O}_{t-1}$ and $v \notin \mathbb{O}_{t-1}$. Follow the cycle $C$ starting from the vertex $v$. If the node $w$ after $v$ in $C$ is not in $\mathbb{O}_{t-1}$, then we have found two nodes in $\mathcal{O}_{t}-\mathbb{O}_{t-1}$, and we are done. So, $w$ is in $\mathbb{O}_{t-1}$. Now, continue traversing $C$ from $w$ to $u$. By Lemma 14 , after applying $\operatorname{BFS}\left(\mathbb{O}_{t-1}, S_{t-1}\right)$, the forest $\mathcal{A}_{S_{t-1}}^{+}\left(\mathbb{O}_{t-1}\right)$ is contained in the routing graph. In fact, the routing graph at this point is exactly $S_{t-1}$, and no nodes change their choice. Hence, all nodes are consistent after applying $\operatorname{BFS}\left(\mathbb{O}_{t-1}, S_{t-1}\right)$. This means that $w$ is not a descendant of $u$ or $v$ in the forest $\mathcal{A}_{S_{t-1}}^{+}\left(\mathbb{O}_{t-1}\right)$; otherwise, $w$ would have $u$ and $v$ (in fact, the $\operatorname{arc}(u, v))$ in its chosen route. So, the only way $C$ can go from $w$ to $u$ is to leave the set of nodes $\mathbb{O}_{t-1}$. But then the next node $y$ on $C$ we reach outside $\mathbb{O}_{t-1}$ satisfies $y \neq v$. Therefore, again, $C$ has two distinct nodes not in $\mathbb{O}_{t-1}$.

It is immediate from Lemma 15 that we can route every packet in $\lfloor n / 3\rfloor$ rounds, and that the network becomes stable in $n$ rounds. Moreover, we can deduce a stronger failure guarantee. We say that round $t$ is a imperfect round if we cannot route every packet. Then there can be at most $\lfloor n / 3\rfloor$ imperfect rounds (note that these may not be consecutive rounds) even if the routing graph is not yet stable.

Theorem 16. There is an activation sequence that routes every packet in $\lfloor n / 3\rfloor$ rounds, gives a stable spanning tree in $n$ rounds, and guarantees that there are at most $\lfloor n / 3\rfloor$ imperfect rounds.

Acknowledgements. We thank Michael Schapira and Sharon Goldberg for interesting discussions on this topic.

\section{References}

[1] Thomas Erlebach, Alexander Hall, Alessandro Panconesi, and Danica Vukadinovic. Cuts and disjoint paths in the valley-free model. Internet Mathematics, 3(3), 2007. 21

[2] Alex Fabrikant and Christos H. Papadimitriou. The complexity of game dynamics: BGP oscillations, sink equilibria, and beyond. In SODA, pages 844-853, 2008. 6

[3] Alex Fabrikant, Umar Syed, and Jennifer Rexford. There's something about MRAI: Timing diversity can exponentially worsen BGP convergence. In INFOCOM, pages 2975-2983, 2011. 20 
[4] Lixin Gao and Jennifer Rexford. Stable internet routing without global coordination. IEEE/ACM Trans. Netw., 9(6):681-692, 2001. 20

[5] Timothy Griffin, F. Bruce Shepherd, and Gordon T. Wilfong. The stable paths problem and interdomain routing. IEEE/ACM Trans. Netw., 10(2):232-243, 2002. 1, 5, 20

[6] Timothy Griffin and Gordon T. Wilfong. An analysis of BGP convergence properties. In SIGCOMM, pages 277-288, 1999. 6, 20

[7] Timothy G. Griffin and Brian J. Premore. An experimental analysis of BGP convergence time. In ICNP, pages 53-61, 2001. 20

[8] John P. John, Ethan Katz-Bassett, Arvind Krishnamurthy, Thomas E. Anderson, and Arun Venkataramani. Consensus routing: The internet as a distributed system. (best paper). In NSDI, pages 351-364, 2008. 21

[9] Howard J. Karloff. On the convergence time of a path-vector protocol. In SODA, pages 605-614, 2004. 20

[10] Richard M. Karp. Reducibility among combinatorial problems. In Complexity of Computer Computations, pages 85-103, 1972. 6

[11] Nate Kushman, Srikanth Kandula, Dina Katabi, and Bruce M. Maggs. R-BGP: Staying connected in a connected world. In NSDI, 2007. 20

[12] Craig Labovitz, Abha Ahuja, Abhijit Bose, and Farnam Jahanian. Delayed internet routing convergence. IEEE/ACM Trans. Netw., 9(3):293-306, 2001. 20

[13] Hagay Levin, Michael Schapira, and Aviv Zohar. Interdomain routing and games. SIAM J. Comput., 40(6):1892-1912, 2011. 21

[14] Zhuoqing Morley Mao, Jennifer Rexford, Jia Wang, and Randy H. Katz. Towards an accurate as-level traceroute tool. In SIGCOMM, pages 365-378, 2003. 21

[15] Dan Pei, Matt Azuma, Daniel Massey, and Lixia Zhang. BGP-RCN: improving BGP convergence through root cause notification. Computer Networks, 48(2):175-194, 2005. 20

[16] Michael Schapira, Yaping Zhu, and Jennifer Rexford. Putting BGP on the right path: a case for next-hop routing. In HotNets, page 3, 2010. 20, 21

[17] John W. Stewart, III. BGP4: Inter-Domain Routing in the Internet. Addison-Wesley Longman Publishing Co., Inc., Boston, MA, USA, 1998. 12, 20

[18] Kannan Varadhan, Ramesh Govindan, and Deborah Estrin. Persistent route oscillations in inter-domain routing. Computer Networks, 32(1):1-16, 2000. 20

[19] Stefano Vissicchio, Luca Cittadini, Laurent Vanbever, and Olivier Bonaventure. iBGP deceptions: More sessions, fewer routes. In INFOCOM, 2012. 2 


\section{Appendix: Interdomain Routing and Model Technicalities.}

The Internet is a union of subnetworks called domains or Autonomous Systems (ASes). The interdomain routing protocol used in the Internet today is called the Border Gateway Protocol (BGP), and it works as follows [17]. For destination $r$ and router $v$, each neighbouring router of $v$ announces to $v$ the route to $r$ that it has chosen and from amongst these announced routes, $v$ chooses the route $\mathcal{P}(v)$ that it ranks highest. The router $v$ then in turn announces to its neighbouring routers its routing path $\mathcal{P}(v)$. This process continues until an equilibrium is reached in which each router has chosen a route and for each router $v$, no neighbour of $v$ announces a route that $v$ would rank higher than its currently routing path. The ranking of routes at a router depends on a number of route attributes such as which neighbour announced the route, how long the route is, and which domains the route traverses. In fact, the ranking of routes at $v$ is a function of $v$ 's traffic engineering goals as well as the Service Level Agreements (SLAs), that is, the economic contracts $v$ has made with its neighbours.

It is well known that BGP can be thought of as a game [5] and that BGP as a game may have no Nash equilibrium [18, 6]. There is now a vast literature studying the conditions under which BGP will or will not have an equilibrium (for example [5, 4]). It has been shown that in a BGP instance, the absence of a structure known as a dispute wheel implies that the BGP instance will have a unique equilibrium [5]. There have been a number of papers analysing the worst-case convergence time of BGP instances having no dispute wheel [9, 3, 16]. There have also been many experimental papers measuring BGP convergence times [12, 7] and papers offering modifications to BGP with the goal of speeding up convergence [15, 11].

However, BGP convergence is only a step towards the ultimate goal of successfully delivering packets to the destination. In fact, routers perform operations simultaneously on two basic levels: (1) on the control plane (i.e., where BGP exchanges routing information with other routers as described above) and (2) on the forwarding plane where routers use the routing information from BGP to forward packets to neighbouring routers towards the packets' ultimate destinations. That is, packets are being forwarded during the time that the control plane is attempting to settle on an equilibrium.

Recall our model in Section 2, We base our idealised routing protocols on BGP and two particularly important attributes that a routers uses to rank its available routes. Firstly, a router might not trust certain domains to handle its packets securely or in a timely fashion, so it may reject routes traversing such unreliable domains. This motivates a (no-go) filtering, which will filter out any route that goes through an undesirable domain (i.e., a domain on the router's no-go filtering list). Secondly, it has been argued that perhaps the most important attribute in how a router $v$ ranks routes is the neighbour of $v$ announcing the route to $v$ [16]. That is, one can think of each router ordering its neighbours and ranking any route from a lower ordered router over any route from a higher ordered router. This is called next-hop routing. Thus, in our protocols, a node ranks routes by first filtering out any route that goes through nodes on its filtering list and then choosing from amongst the remaining routes the one announced by the lowest ordered neighbour (next-hop preference with filtering).

As discussed, to analyse stability, it suffices to consider only the control plane. But, to understand packet routing, we need to understand the interaction between the forwarding and control planes. Thus, we need to incorporate the actions of the forwarding plane into the standard model of the control plane [5]. To do so, some assumptions must be made, particularly concerning the synchronisation between the planes. In setting up a model for a practical problem, it is impor- 
tant to examine how the modelling assumptions relate to reality. So, here we briefly address some technical aspects:

- Synchronisation of the Planes. Observe that, in our model, the control plane and the forwarding plane operate at a similar speed. This assumption is the worst case in that it maximises the rate at which inconsistencies are produced between the nodes routing paths. In practice, updates in the control plane are much slower than the rate of packet transfer.

- Packet Cycling. When a packet gets stuck in a cycle, we will assume that, at the start of the next round, an adversary can position the packet at whichever node in the cycle they wish.

- Fair Activation Sequences. We insist that activation sequences in the control plane are fair in that all nodes update their routes at a similar rate. Clearly, the use of permutations ensures fairness. From the theoretical point of view, fairness is important as it avoids artificially routing packets by the use of unnatural and pathological activation sequences. For example, it prohibits the use of activation sequences that are biased towards nodes in regions where disconnectivities arise and attempts to fix this by "freezing" other nodes until consistency is obtained. Moreover, in practice, routers timings on the control plane are similar.

- Routing in Rounds. The use of rounds (defined by permutations) for routing is not vital and is used for clarity of exposition and to emphasise fairness. Also, packet forwarding is clearly not delayed until the end of a "round" in practice but, again, this is also not needed for the model. The assumption is made as it clarifies the arguments needed in the analyses. For example, forwarding at the end of a round can be shown to be equivalent to forwarding continuously throughout the round with the planes in sync; that is, packets are forwarded immediately and, within a round, the routing path at a node is updated just before the first packet a node sees is about to leave it.

- Route-Verification. Route-verification at the end of the round is our one non-worst case assumption and is not a standard aspect of BGP, albeit one that can be incorporated in a fairly simple fashion by tools such as traceroute or an AS-level traceroute tool such as that described by Mao et al. [14]. Route-verification is the focus of the influential paper of John et al. 8] on consensus routing. It is also used in the theory literature on incentives under BGP [13. Due to the manipulative power provided by unfair activation sequences, it is not hard to simplify our algorithms and omit the route-verification step given the use of unfair activation sequences; see also [16]. It remains an interesting open problem to obtain consistency using fair sequences without route-verification.

- Filtering. In this paper, we assume that each node can apply what is known as import filtering - that is, not accepting certain routes from its neighbours. This implicitly assumes that each node announces its routing path to all of its neighbours. In reality, each node may choose to apply export filtering - that is, it may announce any particular route to only a subset of its neighbours (e.g., in order to assure "valley-free routing" [1]).

Export filtering can be incorporated into our model by allowing for neighbour specific import filtering rules, where a node $v$ can have a filtering list $\mathcal{D}(v, w)$ for each neighbour $w$. Of course, our lower bounds would still hold for this more general model, but it would allow for more special cases to explore. 